\title{
Improved Precoding Method for PAPR Reduction in OFDM with Bounded Distortion
}

\author{
Namitha.A.S \\ Department of Electronics and \\ communication \\ Amrita Vishwa Vidya Peetham \\ Coimbatore,India
}

\author{
Sudheesh.P \\ Department of Electronics and \\ communication \\ Amrita Vishwa Vidya Peetham \\ Coimbatore,India
}

\begin{abstract}
Orthogonal Frequency Division Multiplexing (OFDM) is a very attractive technique for high-data-rate transmission in wireless and wired applications. The main drawback of the OFDM technique is its high peak to average power ratio(PAPR). Several approaches exists to reduce PAPR of OFDM symbols. Precoding is a new method which is having less complexity compared to the other power reduction techniques and also it can reduce PAPR considerably and results in no distortion. Error control coding(convolution coding is used here) can also be introduced along with the Precoding technique in order to improve the bit error rate performance. Clipping and filtering with bounded distortion is a simple method and provides a high PAPR reduction at the cost of signal distortion. we can restrict this distortion within a particular limit using bounded distortion algorithm(BD). In this paper the PAPR reduction is obtained by combining the precoding method and clipping technique with BD i.e. this modified method takes the advantages of two PAPR reduction technique. This paper presents various computer simulation results to verify the effectiveness of this modified precoding method.
\end{abstract}

\section{Keywords}

PAPR; OFDM; SLM; PTS; TR; clipping; Precoding; CFBD.

\section{INTRODUCTION}

OFDM has recently seen rising popularity in wireless applications since it provides an efficient means to mitigate the intersymbol interference (ISI) caused by the channel multipath spread and high data rate transmission. This is a multicarrier technique in which modulating the entire data stream with different subcarriers and each of these subcarriers is orthogonal to each other. An OFDM-based system can provides greater immunity to multipath fading. OFDM has been widely considered for digital communication systems such as wireless local area networks and digital audio/video broadcasting services . It is also being considered for future broadband applications and fourth generation transmission technique. An OFDM transmitter can be implemented by using inverse fast Fourier transform (IFFT) and the output of IFFT block is a time domain signal. The output of IFFT(OFDM signals) have an inherent difficulty that it may exhibit a very high peaks since it is generated by the addition of several independently modulated signal. The power of these large peaks will be very high compared to the average power of the signal. Hence peak to average power ratio is very high which is considered as the major disadvantage of the OFDM technique. These large peaks cause saturation in power amplifiers which is placed at the front end of the transmitter and leads to nonlinear distortions [1]. This paper elaborates on a new technique named Precoding for Peak to Average Power Ratio Reduction

Several PAPR reduction schemes have been proposed to alleviate this problem $[2,3,4,5,6]$. The simplest method of eliminating this high peaks is clipping and filtering method [3]. The OFDM signal is deliberately clipped at a particular threshold value before amplification in this method [5]. The large peaks of OFDM signals occur with a very low probability and hence clipping could be an effective technique for the reduction of the PAPR. However, clipping cause significant in-band distortion and out-of-band noise which will indirectly degrades the bit error rate performance and the spectral efficiency. Filtering is done after clipping in order to eliminate unwanted frequencies caused by the clipping process.

Another solution is to use selective mapping method [13]. The entire data stream is divided into different blocks of $\mathrm{N}$ symbols each. Each block is multiplied with $U$ different phase factors to generate $U$ modified blocks before giving to IFFT block. Each modified block is given to different IFFT block to generate OFDM symbols. PAPR is calculated for each modified block and select the block which is having minimum PAPR ratio. This technique can reduce PAPR considerably. But this technique will increase circuit complexity since it contains several IFFT calculations.

Partial transmit sequence technique (PTS) is another method that has been proposed and studied in the literature [6] . In PTS technique, an input data block of $\mathrm{N}$ symbols is partitioned into disjoint subblocks. The subcarriers in each subblock are multiplied by a phase factor. The phase factors are selected such that the PAPR of the subblocks is minimized. Optimization techniques used to select the phase factors in order to achieve the above objective. Each of the subblocks having the minimum PAPR and hence the combined signal of the different subblocks is having the minimized PAPR.

Tone reservation method of the OFDM signal [9] appears as an attractive solution for reducing the PAPR of OFDM signals. In this method some OFDM subcarriers are reserved. These reserved subcarriers don't carry any information, are only used for reducing PAPR. This method restricts the data vector, and the peak reduction vector to lie in disjoint frequency subspaces. The gradient algorithm is one of the good solution used in this method to reduce PAPR ratio with low complexity. The basic idea of the gradient algorithm has come from clipping. Clipping 
the peak tone to the target clipping level can be interpreted as subtracting impulse function from the peak tone in the time domain. Impulse function is time shifted to the peak tone location, and scaled so that the power of the peak tone should be reduced to the desired target clipping level, when subtracting this impulse function from the original one.

A novel scheme called Precoding of OFDM signals is addressed for the reduction of the peak-to-average power ratio (PAPR) in OFDM systems with less complexity. In Precoding, ,we are multiplying the modulated data of each OFDM block by a precoding matrix before giving to IFFT block .

\section{PAPR IN OFDM}

The entire bit stream that we have to transmit is divided into several blocks of $\mathrm{N}$ symbols each. For a data block $\quad \mathbf{x}=[x 0$, $\left.x 1, \cdots, x_{N-1}\right]$, the OFDM signal $S \mathbf{x}(t)$ can be represented as

$S_{X}(t)=\sum_{k=0}^{\mathrm{n}-1} x_{k} e^{j 2 \pi k t / T}$

where $\mathbf{1} / \mathbf{T}$ is the bandwidth of each subcarrier used for modulation. The PAPR of the transmit data $\mathrm{X}$ is defined as

$\operatorname{PAPR}(X)=\max \frac{P_{X(t)}(t)}{P_{\text {axt }}}, \quad 0 \leq t<T$

Where $P_{X}(t)$ is the instantaneous power of the complex vector $\mathbf{x} \in X$ and it is defined by $P_{X}(t)=\left|S_{X}(t)\right|^{2}$ and $\|x\|^{2}$ is its average power where

$\|x\|^{2}=\frac{1}{T} \int_{0}^{T} P_{x}(t) d t=\sum_{k=0}^{N-1} \mid x_{k} \|^{2}$

Where $\mathrm{P}_{\mathrm{avg}}=\sum_{\mathrm{XEXX}}\|x\|^{2} p(x)$ is the average power of the entire bit stream $X$ that we have to transmit and $p(x)$ is the probability of one data block $x$. The PAPR of the entire data $X$ is given by

$\operatorname{PAPR}_{\max }(X)=\max _{x \in X} \operatorname{PAPR}(x)$

\section{PRECODING TECHNIQUE IN OFDM TRANSMISSION}

Initially the entire data stream is given for base band modulation. In conventional OFDM scheme, we are directly giving the modulated data into IFFT block to generate OFDM signals. In precoded OFDM technique, the modulated data is multiplied with a precoding matrix before IFFT operation. No handshake is needed between the transmitter side and the receiver side. Each entries of the precoding matrix should be carefully designed in such a way to reduce PAPR. The precoding matrix is denoted by $\mathrm{P}$ and is defined as

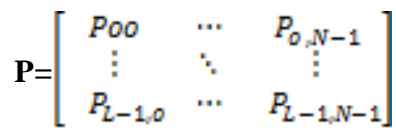

where $P_{i, j} \mathrm{~s}$ are the entries (complex numbers) of this precoding matrix.
A design procedure for good precoding schemes is very important. It is possible to reduce the PAPR of OFDM signals by precoding without destroying the detectability property of the different symbols of the OFDM block [14]. We can use any band efficient modulation like BPSK, QPSK etc.

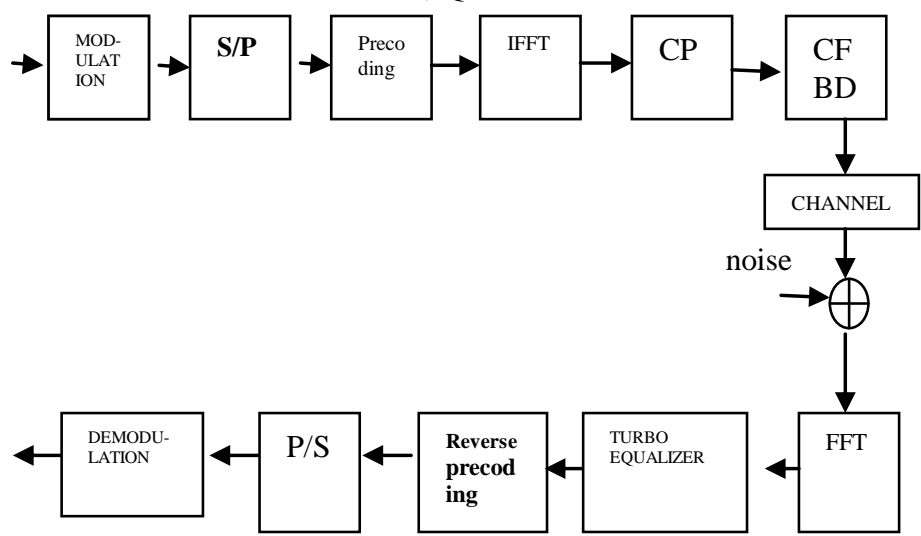

Fig. 1.Block diagram of modified precoding technique of ofdm signals

The transmitter consists of a baseband (complex) modulator followed by a precoder and IFFT. When precoding is not used, the matrix $\boldsymbol{P}$ reduces to an $N \times N$ identity matrix. In precoding, the baseband-modulated stream, with data rate $1 / T s$, is grouped into blocks of length $N$ and it is multiplied by $L X N$ precoding matrix $\mathrm{P}$ given in (5). The total number of subcarriers is represented by $\mathrm{L}$ where $L=N+N p$ where $N p$ is the extra subcarriers used with $0 \leq N p<N$. The base band modulated data of one OFDM block is represented by $\boldsymbol{X}=\left[X_{0}, X_{1}, \ldots\right.$, $\left.X_{N-1}\right]^{\top}$. The precoding process transforms this vector into a new vector of length $L$.

$$
\begin{aligned}
& Y=P X=\left[Y^{O}, Y^{l} \ldots Y^{L-1}\right]^{T} \\
& Y_{i}=\sum_{\mathrm{m}=0}^{\mathrm{N}-1} P_{\mathrm{i}, \mathrm{m}} X_{\mathrm{m}}
\end{aligned}
$$

where i varies from 1 to L-1.. We can convert these signals to low pass equivalent signals and can be written as follows.

$$
\mathrm{x}(\mathrm{t})=\sum_{\mathrm{i}=0}^{L-1} Y_{\mathrm{i}} i e^{j 2 \pi i t / T} \text { where }-T g_{t}<T
$$

where $T=N T s$ is the duration of the OFDM block and $T s$ is the duration of one symbol. Our objective is to minimize the expression of PAPR (2). For that we can reduce the maximum amplitude value of the ofdm symbol. We are not aiming to change the average value. Here, we are trying to relate the PAPR and the entries of the precoding matrix . We can write the equation of PAPR by

$$
P A P R=\max |x(t)|^{2} /\left(E\{|x(t)|\}^{2}\right)
$$

By substituting the value of $x(t)$ in equation (9), we get

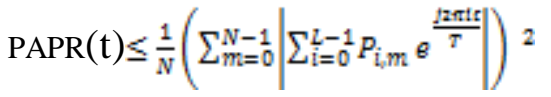

Which is the PAPR of the OFDM signal at a given time instant $t$. By substituting equation (9) in equation 4 , we will get 


$$
\begin{gathered}
\operatorname{PAPR}_{\max } \leq \frac{1}{N} \max _{0 \leq t \leq T}\left(\sum_{m=0}^{N-1}\left|\sum_{i=0}^{L-1} P_{\mathrm{i}, m} e^{j 2 \pi i t / T}\right|\right)^{2} \\
\text { for } m=0,1, \ldots, N-1 .
\end{gathered}
$$

We can define the equation of PAPR in terms of $P_{m}(t)$

$\operatorname{PAPR}(t) \leq \frac{1}{N}\left(\sum_{m=0}^{N-1}\left|P_{m}(t)\right|\right)^{2}$ Where

$p_{m}(t)= \begin{cases}\sum_{i=0}^{L-1} P_{i m} e^{\frac{j 2 \pi i t}{T}} & , 0 \leq t<T \\ 0,0 & \text { otherwise }\end{cases}$

From the above equation, we can see that PAPR is related to the sum of $N$ positive functions within the time interval

$0 \leq t<T$. ie. PAPR is the summation of the different function . Each function is the sum of each column of the precoding matrix. In order to reduce the PAPR , we should have the N different functions that does not having the peak amplitudes at the same time instant .By selecting the different entries of the matrix $P$ such that the different functions are cyclic shifts of each other within the time interval $0 \leq t<T$. To achieve this target, we can impose the following relation between the different functions:

$$
P_{m}(t)=\left\{\begin{array}{cc}
P 0(t-m T s+T), & 0 \leq t<m T s \\
P 0(t-m T s), & m T s \leq t<T
\end{array}\right.
$$

If $p 0(t)$ has only one amplitude peak, then all the other functions will also have one amplitude peak and all the amplitude peaks will not occur at the same time instant since those are cyclic shifts of each other. So this method will surely reduce the peak-to-average ratio of the OFDM signals. We can notice from the above expression that the entries of the precoding matrix are related to each other. All the entries of the different columns of the precoding matrix are directly obtained from the entries of the first column. This indicates that we only need to find the entries of the first column of the matrix $\boldsymbol{P}$. We can design all the entries of the precoding matrix from $\mathrm{p}(\mathrm{t})$ which is the mother function by assuming $\mathrm{p}(\mathrm{t})=\mathrm{p}_{0}(\mathrm{t})$. From the first column we can design all the entries of other columns of the precoding matrix using the equation given below.

$$
\begin{aligned}
P_{i, m} \quad & =P_{i, 0} e^{-j 2 \pi i m / N} \\
& =e^{-j 2 \pi m / N} 1 / T \int_{0}^{T} p(t) e^{-2 \pi i t / T} d t
\end{aligned}
$$

where $p(t)$ can be any of the complex functions within the time interval $0 \leq \mathrm{t}<\mathrm{T}$. The precoding matrix should satisfies the condition $\mathbf{P}^{*} \mathbf{P}=\mathbf{I}$ where $\mathbf{I}$ is the $\mathbf{N} \times \mathrm{N}$ identity matrix, and $\mathbf{P}^{*}$ represents the transpose of the matrix $\mathbf{P}$. By multiplying the $\mathbf{P}^{*}$ matrix at the receiver, we can easily recover the original signal that transmitted. The mother function $P(t)$ is taken here as square root of raised cosine wave

$$
P_{g r c}(f)=\left\{\begin{array}{cl}
T_{g} \operatorname{Sin}\left(\frac{\pi f T_{q}}{2 \beta}\right), & 0<f \leq \frac{\beta}{T_{q}} \\
T_{g_{g}} & \frac{\beta}{T_{g}} \leq f \leq \frac{1}{T_{g}} \\
T_{g} \operatorname{Sin}\left(\frac{\pi\left(f T_{g}-1\right)}{2 \beta}+\frac{\pi}{2}\right), & \frac{1}{T_{g}}<f \leq \frac{1+\beta}{T_{q}}
\end{array}\right.
$$

We know $P_{i, m}=P_{i, 0} e^{-j 2 \pi I m / N}$

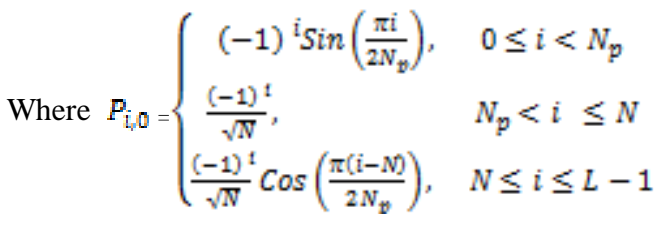

$$
\beta=\frac{N_{\mathrm{WP}}}{N}=\frac{L-N}{N}
$$

where $N_{p}$ is the extra subcarriers used to match with $\mathrm{L}$ point IFFT used in the system to generate OFDM.

\section{CLIPPING AND FILERING WITH BOUNDED DISTORTION (CFBD)}

This is a simplified method of clipping and filtering technique [3] in which we deliberately limiting the amplitude of the time domain signal. The clipped signal $\mathrm{x}_{\mathrm{c}}(\mathrm{t})$ is represented by the relationship

$\mathrm{x}_{\mathrm{c}}(\mathrm{t})=\left\{\begin{array}{rr}A e^{\mathrm{JP}(\mathrm{C}),} & |x(t)|>A \\ x(t), & |x(t)| \leq A\end{array}\right.$

where A is the clipping level, and $\emptyset(t)$ is the phase of input signal. The unwanted frequencies generated because of clipping is called as clipping noise which can eliminate by using a filter after clipper circuit. This filtering operation results in peak regrowth. This can be avoided by $\mathrm{k}$ times repeated clipping [9] which leads to increase in circuit complexity. Instead of repeated clipping, a simplified technique CFBD is mentioned here [10] to eliminate peak regrowth. The main drawback of the repeated clipping and filtering method is its high complexity. In each filtering operation in frequency domain, two FFT calculations are needed. In the simplified method CFBD ,only 3 FFT s are necessary irrespective of $\mathrm{K}$ times repeated operation in iterative clipping method. In order to improve the BER performance as well, the distortion can be bounded as has been proposed in [11] . Block diagram of the CFBD method is shown below.The input signal is limited to the magnitude of level $\mathrm{A}$ in the clipper 
[4]. The clipped signal is subtracted from the original signal in order to compute error signal.

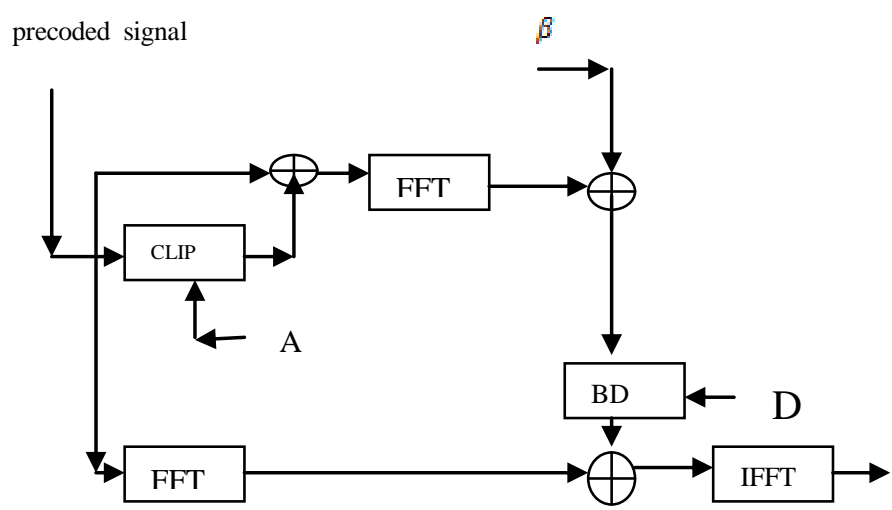

Fig. 2.Block diagram of CFBD method.

This error signal is converted into frequency domain and multiplied by the constant $\beta$ which corresponds to number of iterations chosen for clipping and filtering processes. According to [10], the constant $\beta$ which is multiplied with the error signal is calculated as follows

$\beta \approx \frac{1-(1-\infty)^{\frac{3 k}{2}}}{1-(1-\infty)^{\frac{3}{2}}}$

$\alpha=\frac{2 \sqrt{2}}{\sqrt{(3 \pi)}} \frac{1}{\frac{A}{\sigma}}$

Where $\mathrm{k}$ is the number of repetition of the iterative clipping and filtering method. The real part of OFDM signal $\mathrm{x}(\mathrm{t})$ has normal distribution according to the central limit theorem with variance $\sigma$. The frequency domain error signal is passed through the block ensuring the in-band bounded distortion (BD) after the multiplication with constant $\beta$. The real and the imaginary parts of the error signal are separately treated and limited to a predefined constant $\mathrm{D}$, irrespective of the symbols sent on a particular subcarriers. Some other possible methods are also there in order to reduce the distortion with a bounded predefined constant[11,12]. The error signal with bounded distortion is then subtracted from the original signal where both of these signals are in the frequency domain.

\section{COMBINED PRECODING AND CFBD METHOD}

Precoded OFDM signals can reduce PAPR compared to the conventional OFDM signals. But this method provides a fixed amount of power reduction since the precoding matrix is fixed for all the message signals when the number of subcarriers used for modulation is same. Each OFDM block of $\mathrm{N}$ symbols differ in their magnitude of peak values. Hence the further power reduction according to the message signal is possible by this combined algorithm with CFBD method.

In the combined method of Precoding and clipping,, the PAPR of input OFDM signal is first minimized using Precoding. The precoded OFDM signals is then pass through the
CFBD block for further reduction of PAPR. The distortion caused by the CFBD method is acceptable since it is designed using a control parameter with bounded distortion. Clipping is a simple method compared to many other power reduction techniques. By introducing CFBD (simplified clipping method), circuit complexity does not increases as well as PAPR can be reduced considerably. The PAPR of the OFDM signal is minimized by this combined method more than that of the Precoding method.

\section{SIMULATION RESULTS}

The performance of the different PAPR reduction techniques is simulated and compared. BPSK modulation is taken for simulation. Number of subcarriers is fixed as 64 for IFFT block(64 point IFFT).

The complementary cumulative distribution function (CCDF) of the PAPR is the most commonly used performance measures for PAPR reduction techniques. Cumulative distribution function (CDF) is the probability of a random variable less than a particular value. The complementary CDF (CCDF) is the probability of the obtained PAPR of the data stream exceeds a given threshold. The CCDF is given by

$$
P(P A P R>X)=1-P(P A P R<X)
$$

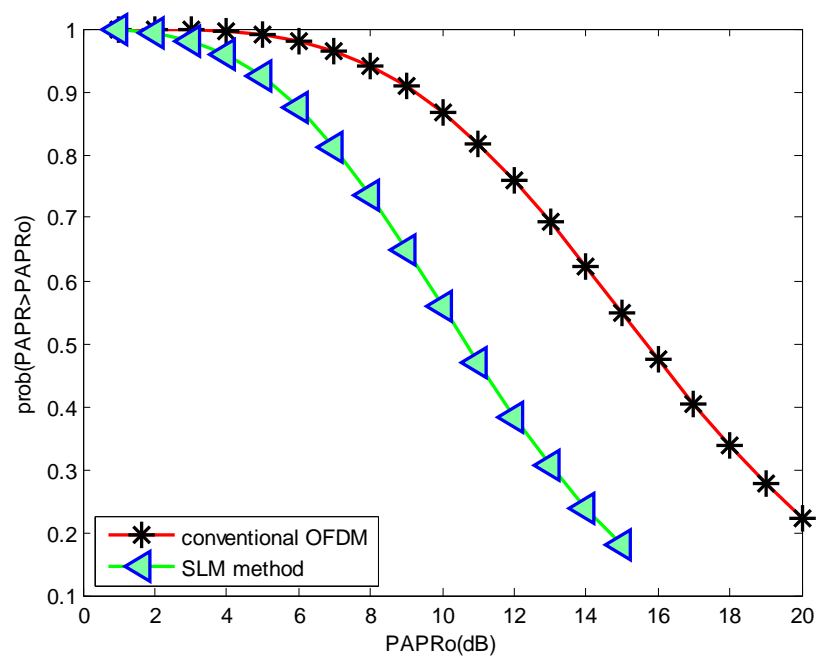

Fig. 3. Complementary distribution function of the PAPR of the OFDM signal with and without SLM technique

Fig.3. shows the CCDFs of PAPR in a BPSK modulated OFDM system with $n=64$ subcarriers .We compare the PAPR statistics of the SLM with conventional scheme where $\mathrm{PAPR}_{0}$ is considered as threshold values in $\mathrm{dB}$.OFDM signals achieve good performance in PAPR reduction using SLM method than conventional OFDM scheme.

For example, if we are taking $10 \mathrm{db}$ as $\mathrm{PAPR}_{0}$.then the probability of obtained PAPR greater than $10 \mathrm{~dB}$ is 85 percentage for conventional OFDM signal and 55 percent for SLM based OFDM technique. 


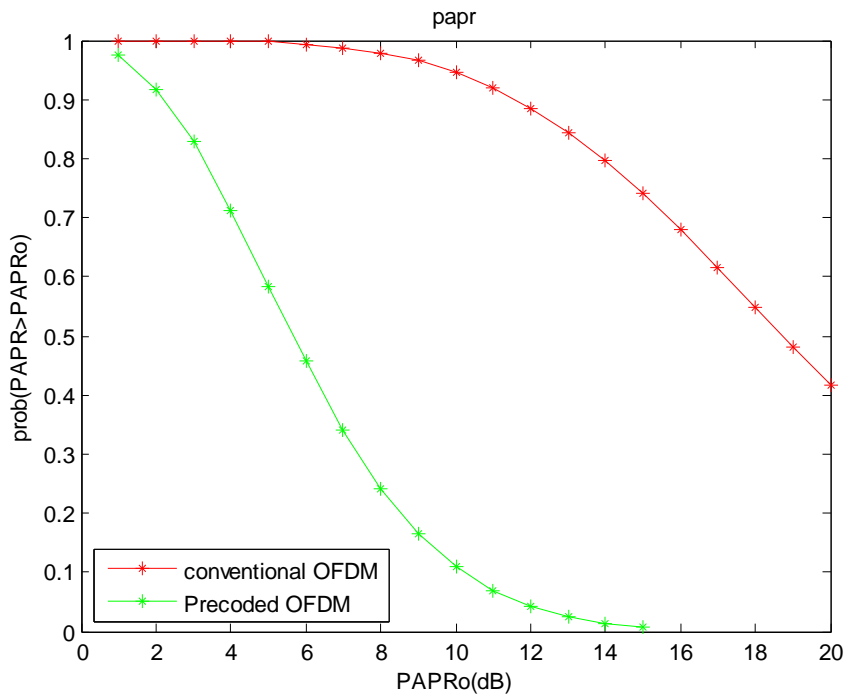

Fig. 5 . CCDF of the PAPR of the OFDM signal using Precoding

The PAPR of the OFDM signal is reduced using Precoding method than that of conventional scheme and it is shown in Fig.5.

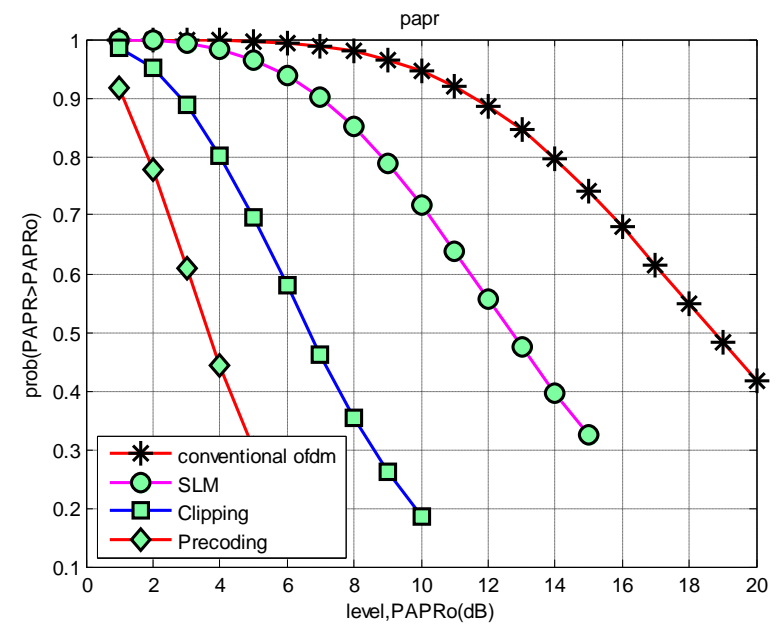

Fig. 6 .Comparison of different PAPR reduction technique

From the Fig.6 ,it is understood that precoding technique have the better performance in PAPR reduction compared to clipping and SLM techniques.

The performance of the combined method is found out on the complementary cumulative distribution function of PAPR. The combined algorithm (Precoding + clipping with BD) is a combination of two approaches to improve the performance of PAPR reduction in OFDM system. Fig.7 shows that the combined algorithm is capable of reducing the PAPR more than that of individual methods.

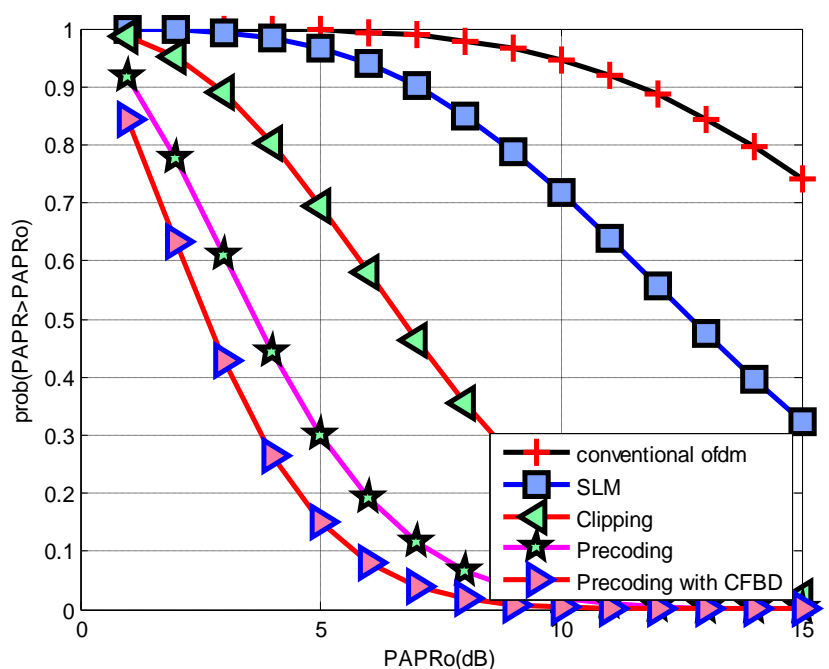

Fig. 7.CCDF of PAPR for the modified precoded OFDM

Fig.8 shows the bit error rate performance of the conventional OFDM system and it is compared with that of Precoded OFDM and combined method of Precoded OFDM with CFBD. Convolution coding is done for improving the BER performance. From the Fig.8, it is understood that Precoded OFDM with CFBD and convolution coding have the best BER performance.

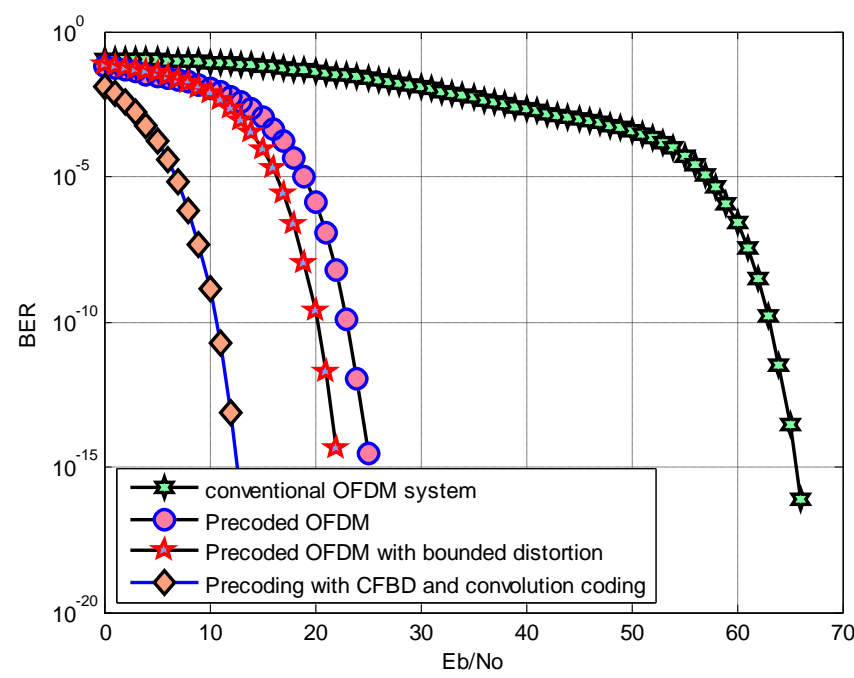

Fig. 8 Bit Error Rate Performance of the modified precoding of OFDM .

\section{CONCLUSION}

A combination of two PAPR reduction methods introduced in this paper. One is based on simplified clipping with $\mathrm{BD}(\mathrm{CFBD})$ and another one is precoding method. CCDF of PAPR plotted for SLM, clipping and PTS techniques. Simulation results show that the Precoded OFDM achieve good performance in PAPR 
reduction. A similar analysis performed for the combined algorithm described above and compared with the individual methods. The modified algorithm shows high performance in PAPR reduction and BER analysis. The precoding method does not increase the circuit complexity compared to other methods. Number of multiplication needed and number of IFFT calculation are comparatively less in this case. In the proposed Precoding method, the OFDM symbols can be detected and decoded at the receiver without the need for sending "side information".

\section{REFERENCES}

[1] C. Rapp, "Effects of HPA-nonlinearity on a 4PSK/OFDMsignal for a digital sound broadcasting system," in Proc. 2nd Eur. Conf. Satell. Commun.Liege, Belgium, Oct. 1991, pp. 179-184.

[2] T. May and H. Rohling, "Reducing the peak-to-average power ratio in OFDM radio transmission systems," in Proc. VTC, Houston, TX, 1999,pp. 2474-2478

[3] G. Ren, H. Zhang, and Y. Chang, "A complementary clipping transform technique for the reduction of peak-toaverage power ratio of OFDM system," IEEE Trans. Consum. Electron., vol. 49, no. 4, pp. 922-926, Nov. 2003

[4] D.Wulich and L. Goldfeld, "Reduction of peak factor in orthogonal multicarrier modulation by amplitude limiting coding," IEEE Trans. Commun., vol. 47, no. 1, pp. 18-21, Jan. 1999.

[5] X. Li and L. J. Cimini, Jr., "Effects of clipping and filtering on the performance of OFDM," in Proc. Globecom, 1997, pp. 1634-1638. [ P. Fan and X.-G. Xia, (2002) The IEEE website. [Online]. Available: http://www.ieee.org/

[6] L. J. Cimini and N. R. Sollenberger, "Peak-to-average power reduction of an OFDM signal using partial transmit sequences," in Proc. IEEE ICC, Vancouver, BC, Canada, 1999, pp. 511-515.
[7] Douillard, M. Jezequel, C. Berrou, A. Picart, P. Didier and A. Glavieux "Iterative Correction of Intersymbol Interference: Turbo Equalization," Euro-pean Transaction on Telecommunications, vol. 6, no. 5, pp 507-511, Sep. Oct.1995.

[8] Sung-Eun Park,Sung-Ryul Yun Jae Yeol Kim,DS Park, Panyuh Joo Samsung "Tone Reservation method for PAPR Reduction scheme ".Elec. 416, Maetan-3dong, Paldal-gu Suwon-si, Gyeonggi-do, Korea, Project :IEEE 802.16 Broadband Wireless Access Working Group <http://ieee802.org/16> 31 ${ }^{\text {st }}$ October 2003.

[9] ARMSTRONG, J.: "Peak-to-average power reduction for OFDM by repeated clipping and frequency domain filtering" Electronics Letters, Vol. 38, No. 5, pp. 246-247, $28^{\text {th }}$ February 2002.

[10] WANG, L., TELLAMBURA, Ch.: "A Simplified Clipping and Filtering Technique for PAR Reduction in OFDM Systems", IEEE Signal Processing Letters, Vol. 12, No. 5, June 2005

[11] URBAN, J., MARSALEK, R.: "OFDM PAPR reduction by simplified clipping and filtering with bounded distortion". In Proc. of the Sixteenth International Electrotechnical and Computer Science Conference ERK 2007, pp. 128-131, November 2007.

[12] DENG, S.-K., LIN, M.-CH." Recursive Clipping and Filtering With Bounded Distortion for PAPR Reduction". IEEE Transactions on communications. 2007, vol. 55, no. 1.

[13] Houshou Chen and Hsinying Liang "A Modified Selective Mapping with PAPR Reduction and Error Correction in OFDM Systems". Dept. of Electrical Engineering and Graduate Institute of Communication Engineering National Chung Hsing University, 250, Kuo Kuang Rd., Taichung 402, Taiwan, 11-15 March 2007

[14] Slimane Ben Slimane, M" Reducing the Peak-to-Average Power Ratio of OFDM Signals Through Precoding" IEEE transactions on vehicular technology, vol. 56, no. 2, march 2007. 